\title{
Characterization of Anti-Myocardial Autoantibodies in Japanese Patients With Dilated Cardiomyopathy
}

\author{
Akiyasu Baba, MD; Tsutomu Yoshikawa, MD*; Masao Chino, MD**; \\ Akira Murayama, $\mathrm{MD}^{\dagger}$; Kazuhiko Mitani, $\mathrm{MD}^{\dagger}$; Susumu Nakagawa, MD ${ }^{\ddagger}$; \\ Isao Fujii, MD*; Megumi Shimada, MD*; Makoto Akaishi, MD; \\ Shiro Iwanaga, MD*; Yasushi Asakura, MD*; Keiichi Fukuda, MD*; \\ Hideo Mitamura, MD*; Satoshi Ogawa, MD*; \\ for the Keio Interhospital Cardiology Study (KICS) Group
}

\begin{abstract}
Few previous reports have comprehensively screened all the anti-myocardial autoantibodies (AMCA) in relation to other clinical profiles in patients with idiopathic dilated cardiomyopathy (IDC), so the present study used both immunohistochemistry (FITC) and immunoblotting (IB) for screening patients with IDC in order to characterize the clinical significance of AMCA. Sera were collected from 100 patients with IDC and age-matched 100 healthy control subjects (CTL). For FITC, an unfixed frozen section of human myocardium was used for the standard indirect immunofluorescence; for IB, total cardiac homogenates of the same myocardium were blotted to serum at 2 sets of dilution (1:200 and 1:10,000). The positive rates of AMCA detection for each method were as follows (IDC vs CTL); $39 \%$ vs $6 \%$ for FITC, $38 \%$ vs $4 \%$ for IB (1:200), and 10\% vs $0 \%$ for IB $(1: 10,000)$. Fifty-nine patients with IDC and 8 CTL were positive for AMCA by either method, and 18 patients with IDC and 2 CTL were positive for AMCA by both methods. IB-positivity at 1:200 was an independent predictor by multiple logistic regression analysis of non-sustained ventricular tachycardias as well as left ventricular enddiastolic diameter and plasma norepinephrine concentration. (Jpn Circ J 2001; 65: 867-873)
\end{abstract}

Key Words: Autoantibodies; Dilated cardiomyopathy; Immunoblotting; Immunohistochemistry

G enetic abnormalities and viral infections are predisposing factors for idiopathic dilated cardiomyopathy (IDC) 1,2 Experimental myocarditis is mediated by alterations in cellular immunity ${ }^{3,4}$ and various autoantibodies are detected in patients with IDC. A recent prospective study examining immunoadsorption therapy of autoantibodies against $\beta_{1 \text {-adrenoceptors has reported that }}$ the therapy improved symptoms and cardiac function in patients with IDC5, and the success with high-dose immunoglobulin $\mathrm{G}$ therapy in patients with myocarditis and acute cardiomyopathy also supports the role of autoimmunity in these conditions. These findings suggest that autoantibodies also have a role in the pathophysiology of IDC, but few previous reports have screened all the anti-myocardial autoantibodies (AMCA) in relation to the other clinical profiles of IDC. Therefore, we used both immunohistochemistry and immunoblotting methods to investigate the clinical significance of the AMCA in the pathophysiology of IDC.

(Received May 14, 2001; revised manuscript received June 18, 2001; accepted July 4, 2001)

Department of Medicine, Kitasato Institute Hospital, Tokyo, *Cardiology Division, Department of Medicine, Keio University School of Medicine, Tokyo, **National Hospital Tokyo Medical Center, Tokyo, †Urawa Municipal Hospital, Urawa, ${ }^{\dagger}$ NKK Hospital, Kawasaki and * Saiseikai Central Hospital, Tokyo, Japan

Mailing address: Akiyasu Baba, MD, Department of Medicine, Kitasato Institute Hospital, 5-9-1 Shirokane, Minato-ku, Tokyo 1088642, Japan. E-mail: baba-a@kitasato.or.jp

\section{Methods}

Study Patients

One hundred patients with IDC were enrolled (79 men, 21 women; mean age, $56 \pm 16$ years, range: $22-85$ years). The diagnosis of IDC was based on the World Health Organization criteria; 7 that is, a dilated and poorly contracting left and/or right ventricle without known cause. Right and left heart catheterization, selective coronary arteriography, left ventriculography, and 2-dimensional echocardiography were performed in all patients within 2 years of enrolment; 51 patients were in New York Heart Association (NYHA) functional class I, 37 in class II, 9 in class III, and 3 in class IV. Another clinical variables were as follows: left ventricular end-diastolic and end-systolic dimensions, 48-103 (mean, 61 \pm 11 ) $\mathrm{mm}$ and 38-93 (mean, 49 \pm 13 ) mm; fractional shortening, 3-31 (mean, 21 \pm 10 ) \%; left atrial di-

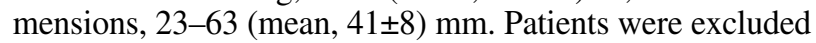
if there was evidence of (1) coronary heart disease on selective coronary arteriography; (2) ischemia during exercise testing; (3) systemic blood pressure $\geq 150 \mathrm{mmHg}$; (4) coexistent systemic or endocrine diseases known to cause left ventricular impairment; and (5) excessive alcohol consumption. Control sera were collected from 100 agematched patients ( 80 men, 20 women; mean age, $56 \pm 15$ years, range: $20-80$ years) who did not have heart diseases (Table 1). All control cases underwent routine physical examination, ECGs, and chest X-ray within 6 months and none had a family history of cardiomyopathies. All patients gave informed consent to participate in the study and the study was approved by the ethics committee of Keio 
Table 1 Characteristics of the Patients With IDC and the Controls

\begin{tabular}{lcc}
\hline \hline & $I D C(n=100)$ & Control $(n=100)$ \\
\hline Age (years) & $56 \pm 16$ & $56 \pm 15$ \\
Male/female & $79 / 21$ & $80 / 20$ \\
NYHA functional class (I/II/III+IV) & $51 / 37 / 12$ & $100 / 0 / 0$ \\
Cardiothoracic ratio (\%) & $58 \pm 6^{*}$ & $42 \pm 5$ \\
LV ejection fraction (\%) & $31 \pm 10$ & $N D$ \\
Plasma norepinephrine (pg/ml) & $425 \pm 290$ & $N D$ \\
Non-sustained ventricular tachycardia (\%) & 46 & ND \\
Anti-myocardial autoantibodies (\%) & $59 *$ & 8 \\
Immunohistochemistry (FITC) (\%) & $39 *$ & 6 \\
Immunoblot $(+)(\%)$ & $38^{*}$ & 4 \\
\hline
\end{tabular}

$*_{p}<0.01$ vs Control. ND, not determined.

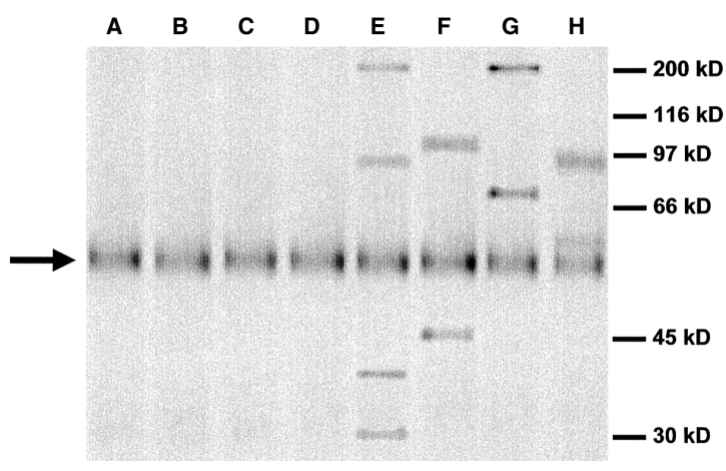

Fig 1. Immunoblotting of anti-myocardial autoantibodies by diluting the patient's serum 1:200 as the primary antibodies. (A,B) Healthy control cases, (C,D) IDC patients without autoantibodies, (E-H) IDC patients with autoantibodies. An arrowhead indicates the presence of immunoglobulin G.

\section{University Hospital.}

\section{Tissue Sources}

Pieces of ventricular myocardium were obtained at autopsy from patients who had not had underlying heart disease and whose blood type was O? Histological examination of the myocardium using conventional hematoxylineosin staining revealed no specific heart muscle disease, necrosis or inflammation. The tissue sample was then processed in 2 different ways. For immunoblotting, tissue was immediately frozen in liquid nitrogen. Whole heart homogenates were boiled in Laemmli's buffer for $3 \mathrm{~min}$, centrifuged for $10 \mathrm{~min}$ at $15,000 \mathrm{G}$ to eliminate aggregates, and stored at $-80^{\circ} \mathrm{C}$ for 1 month before blotting. For immunohistochemistry, tissues were embedded in OCT compound and frozen at $-80^{\circ} \mathrm{C}$.

\section{Sodium Dodecyl Sulfate Polyacrylamide Gel}

\section{Electrophoresis (SDS-PAGE) and Transfer}

Protein (total weight $25 \mathrm{mg}$ ) was separated on $7 \times 8-\mathrm{cm}$ long $10 \% \mathrm{~T}$ (total concentration of acrylamide plus bisacrylamide in g/dl) SDS-PAGE gels with a 3\% T stacking gel. Before electroblotting, the gel and transfer membrane were equilibrated for $15 \mathrm{~min}$ in transfer buffer $(150 \mathrm{mmol} / \mathrm{L}$ Tris, $15 \mathrm{mmol} / \mathrm{L}$ glycine, $10 \%$ ethanol). The proteins were electrophoretically transferred to supported nitrocellulose (Bio-rad, USA) at $500 \mathrm{~mA}$ for $2 \mathrm{~h}$. After transfer, the nitrocellulose was stained with Ponceau red to monitor transfer efficiency and to visualize the protein in each lane. Each lane was separated by pencil marker, and the Ponceau red
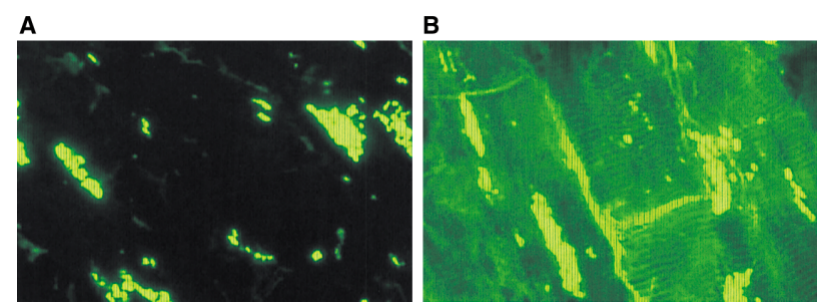

Fig 2. Immunohistochemistry of anti-myocardial autoantibodies by standard indirect immunofluorescence (FITC). IDC patient (A) without autoantibodies, (B) with autoantibodies.

staining was removed by washing with TBS-T $(10 \mathrm{mmol} / \mathrm{L}$ Tris, $150 \mathrm{mmol} / \mathrm{L} \mathrm{NaCl}, 0.1 \%$ Tween $20, \mathrm{pH} 8.0$ ).

\section{Immunoblotting}

The nitrocellulose membranes were soaked in 3\% bovine serum albumin dissolved in TBS-T for $1 \mathrm{~h}$ at room temperature. After blocking, the membrane was cut into strips corresponding to the lanes marked by the pencil. The blots were then incubated for $1 \mathrm{~h}$ in serum samples from patients or controls diluted in TBS-Tat to 1:200 or 1:10,000. After washing 3 times with TBS-T, the blots were incubated for $30 \mathrm{~min}$ at room temperature with appropriate peroxidase-labeled secondary antibody (ie, goat antihuman IgG; Boehringer Mannheim, USA). The protein bands to which the AMCA were bound were visualized using an enhanced chemiluminescence detection system (Amersham Pharmacia, UK). After washing vigorously with TBS-T, blots were incubated with the detection reagent for $1 \mathrm{~min}$ and then exposed to autoradiography film (Kodak, Japan) for 1, 2 and $5 \mathrm{~min}$. The films were developed using an automatic radiograph developer.

\section{Immunohistochemistry}

Immunohistochemical staining of myocardial tissue samples was performed using standard indirect immunofluorescence at 1:10 dilution of the patient's serum on 4-mm unfixed fresh frozen cryostat sections of the myocardium. Fluorescein isothiocyanate (FITC)-labeled goat anti-human $\mathrm{IgG}$ (Cappel, USA) was used as the secondary antibody at 1:50 dilution, and the sections were viewed on an ultraviolet microscope (Olympus, Japan).

\section{Clinical Features of Autoantibodies-Positive Patients}

We compared age, gender, NYHA functional class, left ventricular ejection fraction (LVEF) and LV dimensions, plasma potassium, norepinephrine, renin, atrial natriuretic 
Table 2 Autoantibodies Detected by Immunoblotting and FITC

\begin{tabular}{|c|c|c|c|c|}
\hline & \multicolumn{2}{|c|}{$I D C(n=100)$} & \multicolumn{2}{|c|}{ Control $(n=100)$} \\
\hline & $\operatorname{FITC}(+)$ & FITC (-) & $\operatorname{FITC}(+)$ & $\operatorname{FITC}(-)$ \\
\hline Immunoblot (+) & 18 & 20 & 2 & 2 \\
\hline Immunoblot (-) & 21 & 41 & 4 & 92 \\
\hline
\end{tabular}

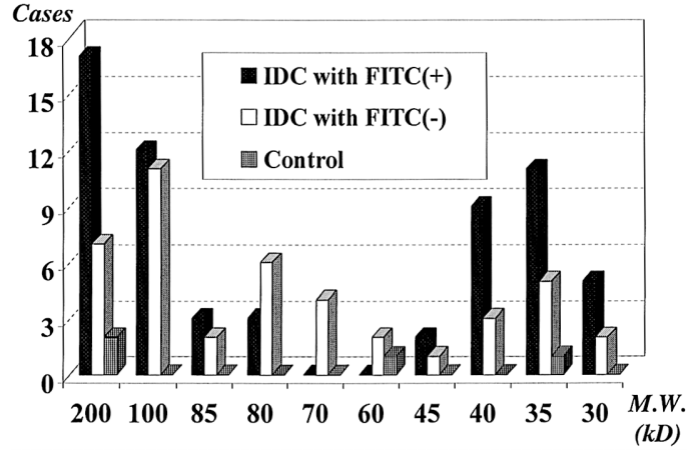

peptide concentrations, and the frequency of premature ventricular contractions (PVC) and ventricular tachycardia (VT) on 24-h Holter monitoring between patients with and those without autoantibodies. Echocardiography was performed using a commercially available probe and system. Holter electrocardiograms were analyzed using a Marquette system, and data were averaged from 2 or more sets of recordings.

\section{Statistical Analyses}

Statistical analysis was performed with StatView 5.0 software (SAS Institute Inc, USA) on a Windows personal computer. Categorical data were analyzed using the chisquare test or Fisher's exact test when appropriate. Unpaired Student's t test was used to compare other continuous variables. All continuous data are expressed as mean \pm SD. Multiple logistic regression analyses were performed in order to determine which factors were significantly associated with the risk of non-sustained VT. Odds ratios (OR) were calculated to measure the degree of association. Statistical significance was defined as $\mathrm{p}<0.05$. The confidence limit chosen was $95 \%$.

\section{Results}

Fig 1 is a representative immunoblot. We defined a sample as positive if the AMCA recognized some proteins other than this IgG. By diluting the sera to 1:200, there were 38 positive cases in the patients with IDC, and 4 cases in the control group (Table 1).

For immunohistochemistry, we carried out the experiment free from primary antibodies as negative control. There was fluorescence around the blood vessels and lipofuscin, even in negative controls (Fig 2A), but the putative myofibril staining pattern was noted in cardiomyocytes from some cases of IDC (Fig 2B). FITC was positive in 39 patients with IDC and in 6 control subjects (Table 1 ).

Fifty-nine patients with dilated cardiomyopathy and 8 controls had some autoantibodies detected by either immunohistochemistry or immunoblot analysis; 18 patients with IDC and 2 control subjects were positive with both methods (Table 2), and 10 of these patients were still posi-
Fig 3. Molecular weight of antigens and frequency of autoantibodies for immunoblotting by diluting 1:200. FITC $(-)$ or FITC $(+)$ indicates patients without autoantibodies or those with autoantibodies by standard indirect immunofluorescence. tive on immunoblot analysis at 1:10,000 dilution. None of the patients with IDC who were negative with FITC despite positive immunoblotting nor the control subjects were positive at 1:10,000 dilution.

Fig 3 shows the molecular weight of antigens detected by the immunolotting method. The number of cases of a high titer of autoantibodies detected at 1:10,000 dilution was 4 for $35 \mathrm{kDa}, 1$ for $40 \mathrm{kDa}, 1$ for $80 \mathrm{kDa}, 2$ for $100 \mathrm{kDa}$, and 2 for $200 \mathrm{kDa}$. The antigens of $200 \mathrm{kDa}$ and $40 \mathrm{kDa}$ were more frequently detected in patients who were positive on FITC than in the negative patients $(200 \mathrm{kDa}, 17(44 \%)$ vs 7 $(11 \%), \mathrm{p}<0.01,40 \mathrm{kDa}, 9(23 \%)$ vs $3(5 \%), \mathrm{p}<0.01)$.

The clinical profiles of the IDC patients in terms of the presence or absence of autoantibodies detected by the 2 different methods are shown in Table 3. For FITC, the positive cases were older than the negative cases, and digitalis glycosides were used more commonly in patients who did not have autoantibodies. For immunoblot analysis, non-sustained VT (NSVT) was more commonly noted in patients with autoantibodies than in those without autoantibodies, but no difference existed in age, gender, NYHA functional class, LV dimensions, plasma potassium concentration, neurohormones, or other medications.

Table 4 summarizes the results of multiple logistic regression analysis, and demonstrates that autoantibodies detected by immunoblot analysis at 1:200 dilution as well as LV end-diastolic dimension and plasma norepinephrine concentration were independent predictors of NSVT. Interestingly, the presence of $100 \mathrm{kDa}$ autoantibody detected by immunoblotting was significantly associated with the risk of NSVT $(\mathrm{OR}=42.07)$.

\section{Discussion}

Anti-myocardial autoantibodies were screened in 100 patients with IDC using 2 different methods. However, the results of these methods was not consistent, and there was the possibility of the presence of autoantibodies to unidentified antigens. Anti-myocardial autoantibodies were not associated with symptoms, cardiac function, or neurohormones, but patients with the complication of NSVT were detected by positive immunoblot analysis. 
Table 3 Clinical Profiles of Patients With IDC With and Without Autoantibodies

\begin{tabular}{|c|c|c|c|c|}
\hline & $\begin{array}{c}\operatorname{FITC}(+) \\
(n=39)\end{array}$ & $\begin{array}{c}\text { FITC }(-) \\
(n=61)\end{array}$ & $\underset{(n=38)}{\operatorname{Immunoblot}(+)}$ & $\begin{array}{c}\text { Immunoblot (-) } \\
(n=62)\end{array}$ \\
\hline Age (years) & $60 \pm 18 *$ & $54 \pm 15$ & $55 \pm 15$ & $57 \pm 14$ \\
\hline Male/female & $31 / 8$ & $48 / 13$ & $28 / 10$ & $51 / 11$ \\
\hline NYHA functional class $(I / I I / I I I+I V)$ & $20 / 15 / 4$ & $31 / 22 / 8$ & $19 / 14 / 5$ & $32 / 23 / 7$ \\
\hline LV ejection fraction $(\%)$ & $32 \pm 9$ & $30 \pm 10$ & $31 \pm 10$ & $30 \pm 10$ \\
\hline LV end-diastolic diameter $(\mathrm{mm})$ & $60 \pm 9$ & $61 \pm 11$ & $61 \pm 12$ & $61 \pm 10$ \\
\hline LV end-systolic diameter ( $\mathrm{mm}$ ) & $48 \pm 13$ & $49 \pm 14$ & $48 \pm 15$ & $49 \pm 12$ \\
\hline Left atrial diameter $(\mathrm{mm})$ & $42 \pm 8$ & $40 \pm 7$ & $40 \pm 7$ & $41 \pm 8$ \\
\hline Plasma potassium $(\mathrm{mmol} / \mathrm{L})$ & $4.1 \pm 0.1$ & $4.0 \pm 0.1$ & $4.1 \pm 0.1$ & $4.1 \pm 0.1$ \\
\hline Plasma norepinephrine $(\mathrm{pg} / \mathrm{ml})$ & $400 \pm 311$ & $435 \pm 280$ & $465 \pm 308$ & $397 \pm 280$ \\
\hline Plasma renin activity $\left(\mathrm{ng} \cdot \mathrm{ml} \mathrm{l}^{-1} \cdot \mathrm{h}^{-1}\right)$ & $6.5 \pm 8.0$ & $7.5 \pm 8.7$ & $5.6 \pm 7.8$ & $8.1 \pm 8.7$ \\
\hline Plasma atrial natriuretic peptide $(\mathrm{pg} / \mathrm{ml})$ & $98 \pm 98$ & $81 \pm 77$ & $88 \pm 75$ & $87 \pm 91$ \\
\hline Atrial fibrillation (\%) & $11(28)$ & $19(31)$ & $11(29)$ & $19(31)$ \\
\hline Premature atrial contractions (/day) & $359 \pm 1,081$ & $542 \pm 1,375$ & $542 \pm 1,291$ & $428 \pm 1,260$ \\
\hline Premature ventricular contractions (/day) & $2,132 \pm 5,177$ & $2,229 \pm 4,239$ & $2,817 \pm 4,610$ & $1,833 \pm 4,573$ \\
\hline Multi form PVCs (\%) & $29(74)$ & $48(79)$ & $31(82)$ & $46(74)$ \\
\hline NSVT (\%) & $16(41)$ & $30(49)$ & $24(63) * *$ & $22(35)$ \\
\hline Frequency of NSVT (/day) & $88 \pm 264$ & $30 \pm 43$ & $36 \pm 60$ & $62 \pm 214$ \\
\hline Maximum run of NSVT (beats) & $9 \pm 7$ & $8 \pm 6$ & $10 \pm 8$ & $8 \pm 5$ \\
\hline \multicolumn{5}{|l|}{ Concomitant medications } \\
\hline Digitalis glycosides (\%) & $18(46) *$ & $46(75)$ & $26(68)$ & $38(61)$ \\
\hline Diuretics (\%) & $17(44)$ & $41(67)$ & $18(47)$ & $40(65)$ \\
\hline ACE inhibitors (\%) & $19(49)$ & $39(64)$ & $19(50)$ & $39(63)$ \\
\hline$\beta$-blockers (\%) & $9(23)$ & $16(26)$ & $9(24)$ & $16(26)$ \\
\hline Antiarrhythmic agents (\%) & $7(18)$ & $13(21)$ & $9(24)$ & $11(18)$ \\
\hline Inotropic agents (\%) & $3(8)$ & $7(11)$ & $5(13)$ & $5(8)$ \\
\hline Nitrates (\%) & $2(5)$ & $4(7)$ & $2(5)$ & $4(6)$ \\
\hline Antiplatelet agents (\%) & $9(23)$ & $15(25)$ & $10(26)$ & $14(23)$ \\
\hline Anticoagulants (\%) & $13(33)$ & $34(56)$ & $13(34)$ & $34(55)$ \\
\hline
\end{tabular}

${ }^{*} p<0.01$ vs FITC (-); **p<0.01 vs Immunoblot (-).

Table 4 Odds Ratio of Occurrence for Non-Sustained Ventricular Tachycardia

\begin{tabular}{|c|c|c|c|}
\hline & Odds ratio & $95 \% C I$ & $p$ value \\
\hline Age $>60$ years & 1.12 & $0.4-3.4$ & $N S$ \\
\hline Gender (male) & 3.10 & $0.8-12.1$ & $N S$ \\
\hline NYHA functional class (II, III, or IV) & 2.35 & $0.8-7.3$ & $N S$ \\
\hline LV ejection fraction $<30 \%$ & 1.23 & $0.4-4.0$ & $N S$ \\
\hline LV end-diastolic diameter $>60 \mathrm{~mm}$ & 4.67 & $1.4-15.4$ & 0.011 \\
\hline Left atrial diameter $>40 \mathrm{~mm}$ & 2.10 & $0.7-6.5$ & $N S$ \\
\hline Plasma potassium $<4.0 \mathrm{mmol} / \mathrm{L}$ & 1.14 & $0.6-2.3$ & $N S$ \\
\hline Plasma norepinephrine $>400 \mathrm{pg} / \mathrm{ml}$ & 12.76 & $3.3-49.5$ & 0.0002 \\
\hline Plasma renin activity $>7.0 \mathrm{ng} \cdot \mathrm{ml}^{-1} \cdot \mathrm{h}^{-1}$ & 1.52 & $0.4-5.6$ & $N S$ \\
\hline Plasma atrial natriuretic peptide $>80 \mathrm{pg} / \mathrm{ml}$ & 3.07 & $0.97-9.7$ & $N S$ \\
\hline Anti-myocardial autoantibodies & & & $N S$ \\
\hline Immunohistochemistry (FITC) & 1.62 & $0.5-5.2$ & $N S$ \\
\hline Immunoblot $(+)$ & 4.44 & $1.4-14.1$ & 0.012 \\
\hline$L V$ end-diastolic diameter $>60 \mathrm{~mm}$ & 4.47 & $1.3-15.2$ & 0.017 \\
\hline Plasma norepinephrine $>400 \mathrm{pg} / \mathrm{ml}$ & 13.10 & $3.8-45.1$ & $<0.0001$ \\
\hline \multicolumn{4}{|l|}{ M.W. of antigens detected by immunoblot $(k D a)$} \\
\hline 200 & 1.25 & $0.01-158.1$ & $N S$ \\
\hline 100 & 42.07 & $1.8-958.9$ & 0.019 \\
\hline 85 & - & - & $N S$ \\
\hline 80 & 0.15 & $0.0-624.7$ & $N S$ \\
\hline 70 & - & - & $N S$ \\
\hline 60 & - & - & $N S$ \\
\hline 45 & - & - & NS \\
\hline 40 & 0.14 & $0.0-14.2$ & $N S$ \\
\hline 35 & 1.17 & $0.1-23.1$ & $N S$ \\
\hline 30 & - & - & $N S$ \\
\hline
\end{tabular}

Aberrations of the immunoreactions, especially the production of various autoantibodies, have been described in patients with IDC? Most research on AMCA has focused on using FITC to detect anti-myosin autoantibodies and there few clinical studies have used immunoblot analysis. The present study had positive rates for each method of
$39 \%$ and $38 \%$, respectively. In a preliminary study using immunoblotting, we confirmed that the $200 \mathrm{kDa}$ and 40 $\mathrm{kDa}$ bands were myosin heavy chain and actin, respectively, and cardiac myosin and actin have been the more common antigens detected using FITC in patients with autoantibodies? 


\section{Clinical Significance of Autoantibodies Determination}

The following 3 points are important when screening for autoantibodies in patients with IDC. First, a positive result will help confirm the diagnosis. Anti-cardiac autoantibodies are rare in congestive heart failure of ischemic etiology ${ }^{10}$ However, because it is common for IDC patients to have autoantibodies against various cardiac proteins, more sensitive examinations will be required for the differential diagnosis. The preliminary study from our laboratory showed that $38 \%$ of the study population had autoantibodies against $\beta$ 1-adrenoreceptors, which were investigated by enzyme-linked immunosorbent assay (ELISA) ${ }^{11}$ but $72 \%$ of them were positive in one of ELISA, immunoblotting, or FITC.

Second, the examination of autoantibodies has some potential for clinical application in the treatment of such patients. Although a prospective study on immunoadsorption therapy reported an improvement in cardiac performance and clinical status in patients with IDC5 it is still unclear which autoantibodies have pathophysiological significance in these patients. Our results from immunoblot analysis indicate that one-third of FITC-negative patients had AMCA, and that the molecular weight of such antigens differs from the well-known myofibrils. It is, therefore, possible that unidentified autoantibodies are present in sera from patients with IDC.

Third, autoantibodies could be a new prognostic indicator in patients with IDC. Early stage IDC patients are likely to be positive on FITC,12 and cardiac autoantibodies are more commonly detected in their symptom-free relatives than in controls ${ }^{13}$ Antibody-positive relatives had larger ventricular dimensions than negative cases, although NYHA class, LVEF, arrhythmias, and neurohormones were not described in those studies. In the present study, patients who were positive on immunoblotting had complex ventricular arrhythmias.

\section{Detection Methods of Anti-Myocardial Autoantibodies}

Many previous studies have reported using various methods to detect AMCA in patients with IDC. Basically, there were 2 different methods for detecting antigen: (1) using total myocardial tissues (eg, FITC, or immunoblotting), and (2) using expected proteins or polypeptides (eg, supposed myofibrils, or synthetic peptides). There are so many proteins in cardiomyocytes that it seems more sensible to use total myocardial tissues when screening for AMCA. Although the sensitivity using total myocardial tissues may be substantially lower than when using pure antigens, it is reported that the frequency of detecting autoantibodies against the major proteins, such as myosin and actin, does not differ with the method used ${ }^{14}$ Accordingly, the present study used total myocardial tissue as the antigen and, moreover, used the same antigen throughout the experiment.

The ELISA technique is highly suitable for screening a large sample size for given autoantibodies and is able to evaluate the titer of autoantibodies, but it is not suitable for detecting various antigens simultaneously or for detecting unknown antigens. To semiquantitatively investigate antibodies using total myocardial tissues, we organized the primary antibodies in our immunoblot analysis into 2 sets of dilution (1:200 and 1:10,000). Because different IgG purification methods could affect the result, we simply used the patient's serum as the primary antibody. A negative control was set up for each blotting, and whole lanes processed similarly until blocking.

A previous study of 52 patients with IDC reported that $46 \%$ of cases had a high titer of IgG AMCA as demonstrated by immunoblotting using 1:200 dilution of the patient's serum ${ }^{15}$ In our series, $38 \%$ of patients with IDC had AMCA ( $\operatorname{IgG})$ using the same method, but the band corresponding to the heat shock protein-60 was not apparent. Racial differences and differences in the myocardial tissue used as an antigen may be responsible for the discrepancy. Despite the heat shock proteins being highly conserved in the presence of heat, inflammatory mediators and various forms of physiologic stress, they were not expressed in the myocardium used as an antigen in the present study. We also note that the molecular weights of the autoantigens detected by us were different from the study on Caucasian subjects, ${ }^{15}$ but in both studies, there was the the same phenomenon of some patient's serum recognizing several different proteins. Based on these observations, it would seem that the autoimmune mechanism is independent of genetic factors in the pathophysiology of IDC.

We did not detect the previously reported putative AMCA, such as those against $\beta$-adrenergic receptors ${ }^{11}$ the adenine nucleotide translocator ${ }^{16}$ or the M7 mitochondrial antigen ${ }^{17}$ This discrepancy may be explained by the fact that enriched membrane and mitochondrial fractions were used in those previous studies, whereas total cardiac homogenates were used in the present study.

Circulating heart-specific autoantibodies have been reported in $26 \%$ of patients with IDC $^{8}$ and in $20 \%$ of their relatives, ${ }^{13}$ using the FITC method. Fukuta et al examined anti-heart antibodies (IgG subclass) in Japanese patients with IDC using the same FITC method as ours! ${ }^{8}$ They used rat myocardium as the antigens for study, and detected autoantibodies in $65 \%$ of IDC patients. In the present study using human myocardium, 39\% of Japanese patients with IDC were found to have AMCA (IgG) by the same dilution method. It is known that myosin heavy chain is the predominant autoantigen recognized by the heart-specific autoantibodies when using FITC? In the 39 FITC-positive cases in our series, $44 \%$ of them had $200 \mathrm{kDa}$ antigens, corresponding to the myosin heavy chain. Another myofibril also appears to be responsible for the positive FITC in Japanese patients with IDC.

A previous report showed an apparent discrepancy between antigens recognized by immunoblot analysis and those recognized by immunofluorescence antibody assay 19 but the extent of this discrepancy was not known. Immunohistochemistry against unfixed frozen antigens was generally less sensitive than immunoblot analysis in which antigens were denatured in boiled Laemmli's buffer, so some investigators have tried the modifications such as heating antigens even for immunohistochemistry against fixed antigens. On the other hand, some antibodies react better to native antigens than to denatured antigens and therefore, there was the difference of epitope modification between FITC and immunoblot in our study, although we used the same myocardial tissues as antigens in both methods. Regarding the immuno-reactions, autoantibodies do not recognize the same epitopes even if those antibodies recognized the same proteins as antigen. The present study indicated that AMCA were positive for both methods in $18 \%$ of patients with IDC, and 10 of these patients had high titers of autoantibodies detected by immunoblot analysis at 1:10,000 dilution. By immunoscreening for the human heart c-DNA library, we are going to determine the 
sequencing of these proteins, but not all antigens will be included in the proteins of myofibrils; in particular, the autoantibodies to the $100-\mathrm{kDa}$ antigen seem to react with sarcolemmal Na-K-ATPase pump.

\section{Relation to Clinical Profiles}

The clinical significance of AMCA is unclear. The autoantibodies were screened using an immunofluorescence technique in a large sample size in Italy, ${ }^{20}$ and although autoantibodies were recognized in $20 \%$ among 91 patients with IDC, the relationship between clinical profile and the autoantibodies was not examined. They reported that the duration of symptoms was significantly shorter in 5 cases with autoantibodies than in those without antibodies among 11 patients with myocarditis. There is another report of AMCA in 37 patients with end-stage heart failure caused by IDC, who were candidates for heart transplantation. ${ }^{12}$ However, despite using the same FITC technique as ours for detecting autoantibodies, none were positive. In the present study, AMCA were detected by FITC in 39\% of 100 Japanese patients with IDC and the autoantibodies detected were those commonly noted in the previous report from Italy ${ }^{20}$ Although differences in the number of patients and their characteristics are possible for the discrepancies between the studies, the present study involving 100 patients was satisfactory as a study for AMCA. Symptoms of congestive heart failure in our study were milder compared with the previous report from Italy 20 and approximately half of the patients in the present study were NYHA class I, compared with $23 \%$ of patients in the previous study. ${ }^{20}$

There are no previous reports examining the relation between clinical profile and autoantibodies detected by immunoblot analysis. ELISA has been used to investigate $\beta$-adrenoreceptor as an antigen ${ }^{21}$ and autoantibodies directed against $\beta$-adrenoreceptors were positive in all of 13 patients with IDC who had mechanical cardiac support for end-stage congestive heart failure. The autoantibody titer decreased as congestive heart failure was stabilized by therapy. In the present study, autoantibodies detected by immunoblotting were independently associated with the presence of NSVT as well as other clinical factors such as LV end-diastolic dimension and plasma norepinephrine concentration. Of these, the unidentified $100-\mathrm{kDa}$ antigen appears to be related to the NSVT in the present study.

\section{Study Limitations}

We could not determine whether all positive cases in the control subjects were false-positive, for we did not perfectly exclude patients with asymptomatic LV dysfunction among the controls.

We did not include patients with ischemic etiology in this study, and did not check if the autoantibodies were specific to the myocardium. Not all AMCA detected by FITC were found to be cardiac specific in our study.

We could not detect autoantibodies against $\beta$-adrenoreceptor by immunoblot analysis in the present study. Because total cardiac tissue was prepared as antigens, our study failed to detect autoantibodies against such minor proteins as ion channels or pumps. Preliminary experiments from our laboratory suggested that immunoprecipitation was necessary for the immunoblotting of $\beta$-adrenergic receptors.

We can not state which method is superior for detecting AMCA. There were various autoantibodies detected by only one method, either FITC or immunoblotting. We speculate that the ideal screening method for known autoantibodies would be protein chips, which expresses each purified antigen in detectable amounts.

Fluctuation of the autoantibody titer as a result of medical treatment remains possible because we performed only single blood sampling in each patient. If autoantibodies were more commonly noted in decompensated heart failure, heart failure per se will be one of the reasons why arrhythmias increased significantly in patients with autoantibodies in the present study.

In summary, we report that $59 \%$ of Japanese patients with IDC had anti-myocardial antibodies detected by either immunoblotting or FITC. Unknown autoantibodies in addition to those previously reported against myofibrils such as myosin and actin were detected. If such unknown autoantibodies were identified by ELISA, the immunological aspect of IDC would become clearer. Although autoantibodies have been considered as an epiphenomenon, it is clinically helpful to identify those that have some pathophysiological effects. In particular, there were some patients with IDC in whom autoantibodies were detected only by immunoblot analysis and who have unknown autoantibodies that are related to the occurrence of arrhythmias.

\section{Acknowledgment}

This study was supported by a Grant-in-Aid for Encouragement of Young Scientists (No.12770376, 2000) from the Japan Society for the Promotion of Science (Dr Baba Akiyasu).

\section{References}

1. Tsubata S, Bowles KR, Vatta M, Zintz C, Titus J, Muhonen L, et al: Mutations in the human delta-sarcoglycan gene in familial and sporadic dilated cardiomyopathy. J Clin Invest 2000; 106: 655-662

2. Badorff C, Berkely N, Mehrotra S, Talhouk JW, Rhoads RE, Knowlton KU: Enteroviral protease 2A directly cleaves dystrophin and is inhibited by a dystrophin-based substrate analogue. J Biol Chem 2000; 275: $11191-11197$

3. Izumi T, Kodama M, Fujiwara M: Cardiac muscle cell damage through autoimmune mechanism: Can cardiac proteins provoke autoimmune myocarditis? Jpn Circ J 1991; 55: 1138-1143

4. Shioji K, Kishimoto C, Nakayama Y, Sasayama S: Strain difference in rats with experimental giant cell myocarditis. Jpn Circ J 2000; 64: $283-286$

5. Muller J, Wallukat G, Dandel M, Bieda H, Brandes K, Spiegelsberger $\mathrm{S}$, et al: Immunoglobulin adsorption in patients with idiopathic dilated cardiomyopathy. Circulation 2000; 101: 385-391

6. McNamara DM, Rosenblum WD, Janosko KM, Trost MK, Villaneuva FS, Demetris AJ, et al: Intravenous immune globulin in the therapy of myocarditis and acute cardiomyopathy. Circulation 1997; 95: 2476-2478

7. Richardson P, McKenna W, Bristow M, Maisch B, Mautner B, O'Connell J, et al: Report of the 1995 World Health Organization/ International Society and Federation of Cardiology Task Force on the Definition and Classification of Cardiomyopathies. Circulation 1996; 93: $841-842$

8. Caforio AL, Bonifacio E, Stewart JT, Neglia D, Parodi O, Bottazzo GF, et al: Novel organ-specific circulating cardiac autoantibodies in dilated cardiomyopathy. J Am Coll Cardiol 1990; 15: 1527-1534

9. Caforio AL, Goldman JH, Haven AJ, Baig KM, McKenna WJ: Evidence for autoimmunity to myosin and other heart-specific autoantigens in patients with dilated cardiomyopathy and their relatives. Int J Cardiol 1996; 54: 157-163

10. Syrigou EI, Konstandoulakis MM, Syrigos KN, Kroubouzou H, Kokotou E, Toutouzas P: Non-specific autoantibodies in cardiomyopathies and myocardial infarction. J Clin Lab Immunol 1995; 46: $155-162$

11. Magnusson Y, Marullo S, Hoyer S, Waagstein F, Andersson B, Vahlne A, et al: Mapping of a functional autoimmune epitope on the beta 1-adrenergic receptor in patients with idiopathic dilated cardiomyopathy. J Clin Invest 1990; 86: 1658-1663 
12. de Leeuw N, Melchers WJ, Ruiter DJ, Caforio AL, Balk AH, de Jonge N, et al: Autoimmune markers are undetectable in end stage idiopathic dilated cardiomyopathy. J Clin Pathol 1999; 52: 739-743

13. Caforio AL, Keeling PJ, Zachara E, Mestroni L, Camerini F, Mann $\mathrm{JM}$, et al: Evidence from family studies for autoimmunity in dilated cardiomyopathy. Lancet 1994; 344: 773-777

14. Goldman JH, Keeling PJ, Warraich RS, Baig MK, Redwood SR, Dalla Libera L, et al: Autoimmunity to alpha myosin in a subset of patients with idiopathic dilated cardiomyopathy. Br Heart J 1995; 74: $598-603$

15. Latif N, Baker CS, Dunn MJ, Rose ML, Brady P, Yacoub MH: Frequency and specificity of antiheart antibodies in patients with dilated cardiomyopathy detected using SDS-PAGE and western blotting. J Am Coll Cardiol 1993; 22: 1378-1384

16. Schultheiss HP, Bolte HD: Immunological analysis of auto-antibodies against the adenine nucleotide translocator in dilated cardiomyopathy. J Mol Cell Cardiol 1985; 17: 603-617

17. Klein R, Maisch B, Kochsiek K, Berg PA: Demonstration of organ specific antibodies against heart mitochondria (anti-M7) in sera from patients with some forms of heart diseases. Clin Exp Immunol 1984; 58: $283-292$

18. Fukuta S, Yoshinaga T, Yamakawa K, Kimura Y, Kusukawa R: Dilated cardiomyopathy with special reference to humoral immunity. Jpn Circ J 1992; 56: 1073-1080

19. Fedorko DP, Emery DD, Franklin SM, Congdon DD: Evaluation of a rapid enzyme immunoassay system for serologic diagnosis of Mycoplasma pneumoniae infection. Diagn Microbiol Infect Dis 1995; 23: $85-88$

20. Caforio AL, Bauce B, Boffa GM, De Cian F, Angelini A, Melacini $\mathrm{P}$, et al: Autoimmunity in myocarditis and dilated cardiomyopathy: Cardiac autoantibody frequency and clinical correlates in a patient series from Italy. G Ital Cardiol 1997; 27: 106-112

21. Muller J, Wallukat G, Weng YG, Dandel M, Spiegelsberger S, Semrau S, et al: Temporary mechanical left heart support: Recovery of heart function in patients with end-stage idiopathic dilated cardiomyopathy. Herz 1997; 22: 227-236 